\title{
Automatic Enhancement of Low Light Level Image on the Basis of a Gaussian Mixture Model
}

\author{
Liju Yin*, Tingdong Kou, Xuan Wang, Guofeng Zou, Jinfeng Pan, Zhongshang Zhu \\ Zhangdian Shandong University of Technology \\ Shandong, 255000, China
}

${ }^{*}$ Corresponding author's email: LJYIN72 [AT] 163.com

\begin{abstract}
As the first medium to transmit information under a low light level environment. The low light level image is needed in hot-light image imaging technology. The quality of the image will be reduced given the influence of external factors. For example, a sampled image may become blurry. This paper proposes a method for automatic enhancement of low light level image on the basis of a Gaussian mixture model. First, the histogram of the image is modeled with a Gaussian mixture model that is solved by the expectation maximization algorithm of accelerated convergence. The histogram is then partitioned according to the intersection of each cluster. Finally, the mapping relationship of the cluster to which the output image belongs is ascertained and the final enhancement image is obtained. This algorithm can be used to identify the optimal number of clusters and accelerate the convergence speed of the algorithm. Objective evaluation of the Laplace operator value, as well as the grayscale average gradient and contrast (Tab. 1), indicates that the algorithm effectively improves image contrast while maintaining the details of the image.
\end{abstract}

Keywords - Low light level image enhancement; Gaussian mixture model; EM algorithm; robust; histogram

\section{INTRODUCTION}

The development of low light level imaging technology is growing fast given the increasing demand for low light information. Products made from low light level imaging technology have been used in many aspects of our lives. Examples include low light level night vision devices and medical imaging. External noise and other factors reduce the contrast of the output image during the practical application of low light level imaging technology, thereby resulting in low image quality, which affect the subjective observation of the output image or practical usage of this technology ${ }^{[1]}$. Low image quality greatly minimizes the degree of target recognition ${ }^{[2]}$. Thus, light-level image enhancement is crucial in actual application. Thus, we studied a model for enhancing low light level image.

Many algorithms can be applied for low light level image enhancement. For example, histogram equalization ${ }^{[3]}(\mathrm{HE})$ has been widely used in various image enhancement techniques to improve image contrast, especially when contrast is low. This method could facilitate uniformity in the gray distribution of the image. However, the application of HE means that a few gray levels are compressed and the number of gray scales is over-stretched. This process results in reduction of background noise contrast and some useful signals. Thus, HE is not universally used. In recent years, the application of histogram equalization algorithm to study image enhancement has achieved certain results, such as, Contrast Limited Adaptive Histogram Equalization ${ }^{[4]}$ (CLAHE). The algorithm limits local contrast enhancement by limiting the height of the local histogram, thereby limiting noise amplification and local contrast over-enhancement. However, in the boundary 
area of the gray-scale mutation, a lot of noise is generated. In places where the grayscale is particularly concentrated, the output image may be too bright or too dark.

Given the shortcomings of HE and CLAHE, we proposed an optimized automatic enhancement algorithm for low light level image on the basis of the Gaussian mixture model. The Gaussian mixture model models the histogram. An accelerated convergence EM algorithm based on robustness ${ }^{[5]}$ is used to fit the histogram. We used clusters of 2 to 6. Only the histogram data was processed to increase processing speed. The algorithm performs well for image denoising and local detail processing. The proposed algorithm could effectively maintain the details of the image and improves the contrast of the micro-light image.

The remainder of this paper is organized as follows. Sect. 2 provides a detailed presentation of the proposed measurement matrix optimization method. Sect. 3 presents the simulation results. Sect. 4 presents the conclusion.

\section{ESTABLISHMENT OF THE ENHANCED ALGORITHM MODEL}

The Gaussian mixture model consists of Gaussian clusters with different parameters, each of which has its own mean, variance, and weighting coefficients. A Gaussian mixture model can divide and stretch parts of the human eye that are not easily distinguishable. Thus, it can improve not only the image details, but also the contrast of the image ${ }^{[6]}$.

The dynamic range of the input image $\mathrm{X}$ is assumed to be $\left[x_{d}, x_{w}\right]$. The dynamic range of the output image $\mathrm{Y}$ is given as $\left[y_{d}, y_{u}\right]$.

\subsection{Histogram fitting}

$\mathrm{N}$ Gaussian clusters can be used to model the histogram. The probability density function $\mathrm{P}\left(\mathrm{x} \mid \theta_{n}\right)$ calculated from the gray value of the image is a Gaussian mixture distribution that contains $\mathrm{N}$ clusters.

$$
\mathrm{P}(x \mid \theta)=\sum_{i=1}^{N} \alpha_{i} \phi\left(x \mid \theta_{i}\right) .
$$

In Equation (1), $\Phi\left(\mathrm{x} \mid \theta_{i}\right)$ is the probability density of the image grayscale level, which satisfies

$$
\phi\left(\mathrm{x} \mid \theta_{i}\right)=\frac{1}{\sqrt{2 \pi} \sigma_{i}} e^{-\frac{\left(x-\mu_{i}\right)^{2}}{\mathrm{~s} \sigma_{i}^{2}}} .
$$

$\alpha_{i}$ is the prior probability of the $i$ th cluster, which satisfies $\sum_{i=1}^{N} \alpha_{i}=1$ and $\alpha_{i} \in(0,1)$.

The EM algorithm is used to estimate the number of Gaussian clusters and the parameters of each curve in the input image. The number of clusters is selected when $\zeta(x \mid \theta)$ (Equation [4]) changes minimally.

\subsection{Solution and Analysis of the Gaussian Mixture Model}

The Gaussian mixture model is one of the most important modeling approaches. The image enhancement method based on the Gaussian mixture model is a widely used model and an important technique in the field of image processing ${ }^{[7]}$.

\subsubsection{Solution of the Gaussian Mixture Model}

Solving the Gaussian mixture model involves the equation below:

$$
\mathrm{P}\left(x \mid \theta_{n}\right)=\sum_{n=1}^{N} \alpha_{n} \frac{1}{\sqrt{2 \pi} \sigma_{n}} e^{-\frac{\left(x-\mu_{n}\right)^{2}}{2 \sigma_{n}^{2}}} .
$$


$\mathrm{P}\left(x \mid \theta_{n}\right)$ represents an approximation of the probability density of the image histogram and $\mathrm{N}$ represents the number of clusters. $\mu$ and $\sigma^{2}$ represent the mean and variance of the nth clustering level. $\mathrm{P}\left(x \mid \theta_{n}\right)$ is the weighted coefficient of the $n$th cluster. $\mu$ is the grayscale average of the image and $\sigma^{2}$ is the grayscale variance of the image.

The ultimate goal of solving the Gaussian mixture model is to obtain the optimal image gray mean and variance.

Fitting function $\zeta(x \mid \theta)$ in the $\mathrm{FJ}$ algorithm is used ${ }^{8}$; continuous update of the clustering parameters is performed by iteration until $\zeta(x \mid \theta)$ stabilizes and the optimal number of clusters is ascertained.

$$
\zeta(x \mid \theta)=\log P(x \mid \theta) .
$$

The data to be processed is $\mathrm{X}=x_{1}+x_{2}+\cdots+x_{L} . L$ is the maximum grayscale level of the image. Use of the accelerated convergence EM algorithm to process the histogram data not only saves storage space, but also reduces the number of iterations. The steps are as follows.

E-step: Calculate the expected value of a likelihood estimate using data $x$ and current estimate $\theta^{k}$. Identify $\xi_{j k}$ through Equation (3). The final expected function is obtained by Equation (4).

$$
\xi_{j k}=\frac{\alpha_{k} \times \phi\left(x_{j} \mid \theta^{k}\right)}{\sum_{k=1}^{K} \alpha_{k} \times \phi\left(x_{j} \mid \theta^{k}\right)} \text {. }
$$

$\mathrm{Q}\left(\theta, \theta^{k}\right)=\sum_{k=1}^{K} n_{k} \times \log \alpha_{k}+\sum_{j=1}^{N} \xi_{j k} \times\left[\log \left(\frac{1}{\sqrt{2 \pi}}-\log \sigma_{k}-\frac{\left(x_{j}-\mu_{k}\right)^{2}}{2 \sigma_{k}^{2}}\right)\right]$.

$n_{k}=\sum_{j=1}^{N} \xi_{j k}, \sum_{k=1}^{K} n_{k}=N$

$\xi_{j k}$ is the probability that the $j$ th part of the observation data comes from the $k$ th cluster model under the current model parameters.

M Step: Find $\theta^{k}$ that can produce the largest $Q\left(\theta, \theta^{k}\right)$.

1. Obtain the derivative of the $\mathrm{Q}$ function,

$$
\frac{\partial \mathrm{Q}\left(\theta_{0} \theta^{k}\right)}{\partial \theta}=g\left(\theta^{k}\right)
$$

2. If $\left\|g\left(\theta^{k}\right)\right\|=0$, stop calculating. $\theta^{k}$ is for the desired value. Otherwise, proceed to 3 .

3. If $\left\|g\left(\theta^{k}\right)\right\|<\alpha \boldsymbol{r}_{k}$, then take the resulting value to satisfy $\left\|g\left(\theta^{k}\right)\right\| \leq \boldsymbol{r}_{k+1}<\alpha \boldsymbol{r}_{k}$. 
Proceed to 5. Otherwise, proceed to 4.

5. Let $r_{k+1}=r_{k}$, Take the step length of the EM algorithm $\Delta \theta^{(\mathrm{k})}$, and let $\theta^{(\mathrm{k}+1)}=\theta^{\mathrm{k}}+\Delta \theta^{\mathrm{k}}$. $x_{j k}$ is updated by the Symmetric Rank 1 Correction equation, $x_{j k}=x_{j k}^{i+1}+\frac{\left(\Delta_{i}-x_{j k}^{i} \delta^{t}\right)\left(\Delta_{i}-x_{j k}^{i} \delta^{t}\right)^{T}}{\left(\Delta_{i}-x_{j k}^{i} \delta^{t}\right)^{T} \delta^{t}}$.

$$
\Delta_{i}=g\left(\theta^{i+1}\right)-g\left(\theta^{i}\right), \delta_{i}=\theta^{i+1}-\theta^{i} .
$$

6. Let $\mathrm{k}=\mathrm{k}+1$. Return to 1 .

$\mu, \sigma^{2}$ and $\alpha$ are calculated by the following equations:

$$
\begin{gathered}
\sigma_{k}^{2}=\frac{\sum_{j=1}^{N}\left(y_{j}-\mu_{k}\right)^{2}}{\sum_{j=1}^{N} \xi_{j k}} . \\
\mu_{k}=\frac{\sum_{j=1}^{N} \xi_{j k} x_{j k}}{\sum_{j=1}^{N} \xi_{j k}} . \\
\alpha_{k}=\frac{\sum_{j=1}^{N} \xi_{j k}}{N} .
\end{gathered}
$$

Repeat the E, M steps until the parameter iteration is stable, and finally obtain the approximation histogram,

$$
H(x)=M \times \sum_{n=1}^{N} \phi\left(x \mid \theta_{n}\right) \alpha_{n} .
$$

$M$ is the total number of pixels. $\mathrm{H}(\mathrm{x})$ is the number of pixels corresponding to the gradation value $\mathrm{x}$.

\subsubsection{Parameters and Processes for Enhanced Model}

High number of clusters and improved fitting effect and image enhancement effect are optimal conditions for histogram enhancement ${ }^{10}$. However, too many clusters can affect the speed of operations. To achieve the desired effect for both processing speed and enhancement effect, this paper sets the number of clusters at $\mathrm{k}=3 \sim 6$. Convergence function $\zeta(x \mid \theta)$ takes the value of $10^{-6}$ and generally iterates about 100 times. The algorithm flow of this model is shown in Fig. 1. 


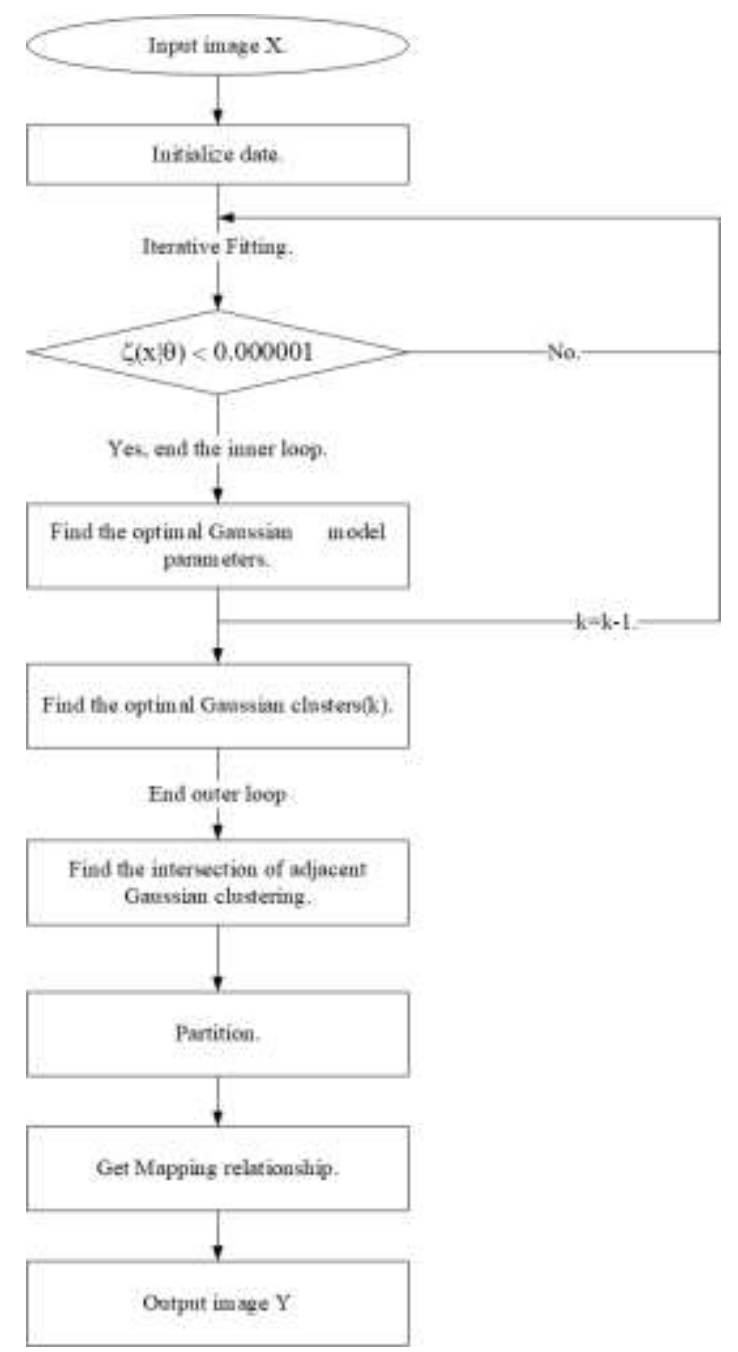

Figure 1: Flowchart of the algorithm

\subsection{Partition}

Meaningful intersections can be obtained by mathematical calculation. The intersection of Gaussian clusters $\theta_{n \text { and }} \theta_{\mathrm{m}}$ can be obtained by Equation (9).

$$
\alpha_{n} \phi\left(x \mid \theta_{n}\right)=\alpha_{m} \phi\left(x \mid \theta_{m}\right)
$$

From Equation 11, we identify all intersections of the Gaussian cluster, but the meaningful intersections are ignored. A meaningful intersection is an intersection of adjacent clusters and must remain within the dynamic range of the gray level.

This cluster must satisfy the following equation:

$\alpha_{n} \phi\left(x_{m, n}^{k} \mid \theta_{\mathrm{m}}\right)=\alpha_{m} \phi\left(x_{m, n}^{k} \mid \theta_{n}\right), \alpha_{n} \phi\left(x_{m, n}^{k} \mid \theta_{\mathrm{n}}\right)>\alpha_{t} \phi\left(x_{m, n}^{k} \mid \theta_{\mathrm{t}}\right)$.

$x_{m, n}^{k}$ represents the $k$ th intersection of the $m$ th cluster and the nth cluster. $\forall \theta_{t} \neq\left\{\theta_{m}, \theta_{n}\right\}$.

The dynamic range $\mathrm{x}$ of the gray level is partitioned by an intersection point, namely,

$x=\left[x^{d}, x^{1}\right] \bigcup\left[x^{1}, x^{2}\right] \bigcup \ldots \cup \ldots \cup \ldots \cup\left[x^{(N-1)}, x^{s}\right]$. 
In the above equation, $x^{d}=x_{d}, x^{s}=x_{u}$.

$x^{k}$ is the intersection of clusters and satisfies $x^{d}<x^{1}<x^{2}<\ldots<x^{(N-1)}<x^{s}$.

\subsection{Mapping}

The essence of grayscale mapping is to map one gray value to another gray value. An image that contains all the new gray values represents the enhanced image as the output.

First, we find the mapping range between the input image and the output image. The cumulative probability density function of the input image is as follows:

$$
F(x)=\int_{-\infty}^{x} P(x) d x=\int_{-\infty}^{x} \sum_{k=1}^{N} \alpha_{k} \phi\left(x \mid \theta_{k}\right) d x .
$$

Each sub-interval of the $\mathrm{x}$ dynamic range is mapped to the corresponding $\mathrm{y}$ sub-interval. The mapping relationship weight coefficient of the interval is determined by $\mathrm{b}_{\mathrm{k}} . b_{k} \in[0,1] . b_{k}$ must satisfy $\sum_{k=1}^{N} b_{k}=1$.

$$
b_{k}=\frac{F\left(x^{k-1}\right)-F\left(x^{k}\right)}{\sum_{i=1}^{K} F\left(x^{i-1}\right)-F\left(x^{i}\right)}
$$

$y^{k}=y_{d}+\left(y_{u}-y_{d}\right) \sum_{i=1}^{k-1} b_{i}$

$y^{k+1}=y^{k}+b_{k}\left(y_{u}-y_{d}\right)$

Equations (15) and (16) can guarantee the coverage of the mapping.

$\left[y_{d}, y_{u}\right]=\left[y_{d}, y^{1}\right] \cup\left[y^{1}, y^{2}\right] \cup \ldots \cup\left[y^{N-1}, y_{u}\right] y_{d}=0, y_{u}=255$

Then, the corresponding Gaussian cluster parameters in the output image are obtained. The cumulative probability density function of the input image is retained in the output image. That is,

$$
\begin{aligned}
& F_{k}\left(x^{k}\right)=F_{k} \cdot\left(y^{k}\right), \\
& F_{k}\left(x^{k+1}\right)=F_{k}\left(y^{k+1}\right) .
\end{aligned}
$$

Therefore, the Gaussian parameters of the image can be solved by Equation (16)-(19).

$$
\begin{aligned}
\mu_{k}^{\prime}= & \frac{\left(\frac{x^{k}-\mu_{k}}{x^{k+1}-\mu_{k}} y^{k+1}-y^{k}\right)}{\left(\frac{x^{k}-\mu_{k}}{x^{k+1}-\mu_{k}}-1\right)}, \\
\sigma_{k}^{\prime}= & \frac{\left(y^{k}-\mu_{k^{\prime}}\right)}{x^{k+1}-\mu_{k}} \sigma_{k} .
\end{aligned}
$$

$\mu_{\kappa}$ is the average of the $k$ th cluster of the original image. $\mu_{k}^{\prime}$ is the average of the $k$ th cluster of the mapped image. 
$\sigma_{k}$ is the variance of the $k$ th cluster of the original image. $\sigma_{k}^{\prime}$ is the variance of the $k$ th cluster of the mapped image.

Mapping relationship is calculated by using the obtained optimal Gaussian parameters. The mapping function is ascertained by weighting the probability density of the clusters in the Gaussian mixture model.

$y=\sum_{k=1}^{N}\left(\left(\frac{x-\mu_{k}}{\sigma_{k}}\right) \sigma_{k}^{\prime}+\mu_{k}^{\prime}\right) \alpha_{k}$.

In the above equation, $\mathrm{x}$ is the input image and $\mathrm{y}$ is the output image after mapping.

Figure 2 shows the best histogram fitting effect when the number of clusters of the Gaussian mixture model of Figure 6 is 5. At this point, six meaningful intersections (black circles) exist, which ensure that a Gaussian cluster plays a dominant role in each sub-interval.

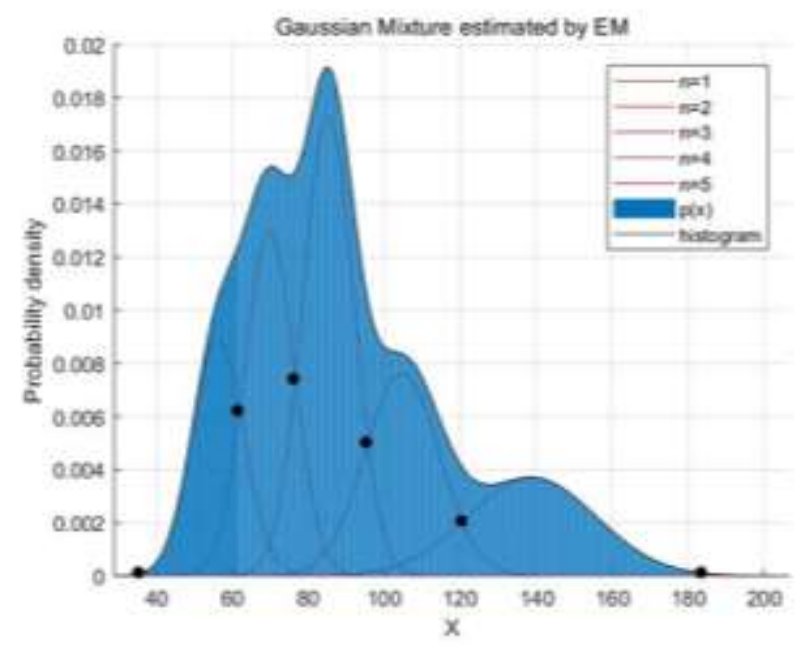

Figure 2: The best fitting result $(\mathrm{k}=5)$ 


\section{EXPERIMENTAL RESULT}

Our laboratory designed a micro-light imaging optical platform to study the enhancement of low light level images. Figure 4 shows the image obtained from the optical platform. The platform mainly consists of the MPPC detector, a LSW101 tungsten light source, a wide-range micro-illuminometer, computer, camera, optical shading cloth, optical fiber, and cable that connects each device. The MPPC detector is essentially a solid-state semiconductor photodetector that is composed of several avalanche photodiodes (Geiger-Model APD) operating in the Geiger mode. MPPC has low crosstalk, low pulse, and low dark count. The noise produced when the APD device is not multiplied is mainly shot noise, thermal noise, and 1/f noise ${ }^{10,11}$. APD is in the Geiger mode when the reverse bias voltage of the APD is higher than the breakdown voltage. Most noise is avalanche excess noise ${ }^{12}$. Avalanche excess noise is similar to shot noise, which is a kind of white noise. In this paper, white noise is used as the main noise object to study the low light level image. The block and physical diagrams of the MPPC low light level imaging optical platform are shown in Figure 3.

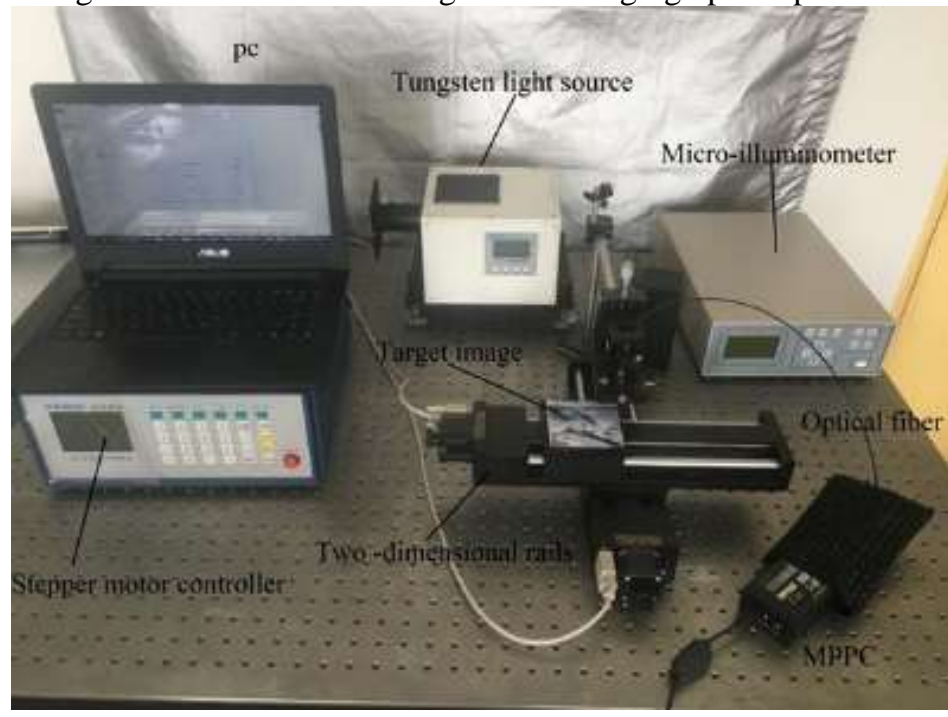

Figure 3 Optical platform.

\subsection{Subjective Analysis}

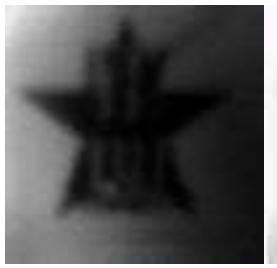

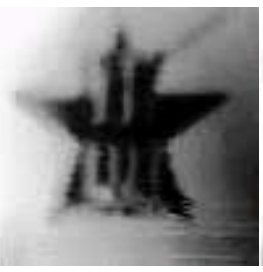

(a)

(b)

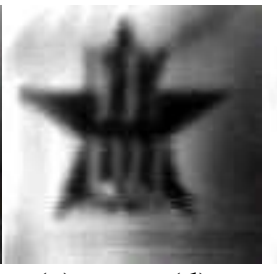

(d)

(c)

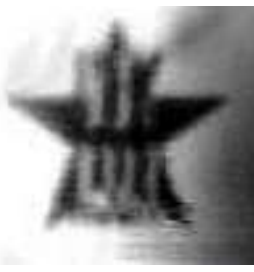

Figure 4 Enhancement results. (a) Original image; (b) HE; (c)CLAHE; (d) The proposed method

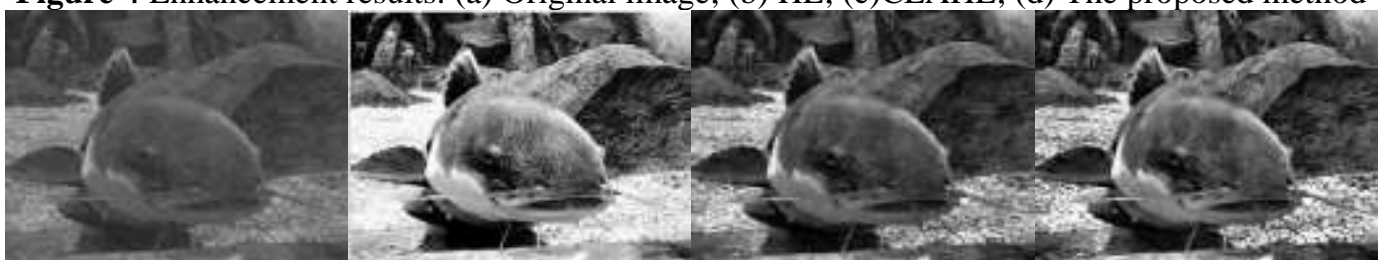

(a) (b) (d)

Figure 5 Enhancement results. (a) Original image; (b) HE; (c)CLAHE; (d) The proposed method

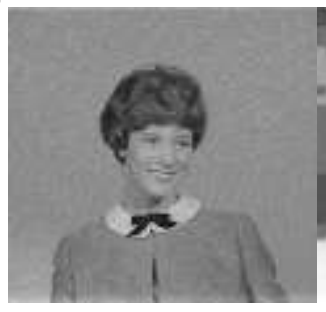

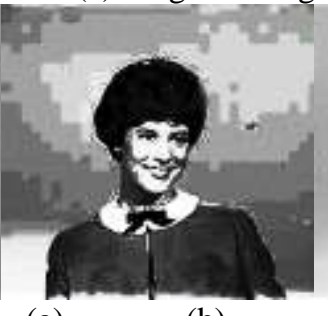

(a)

(b)

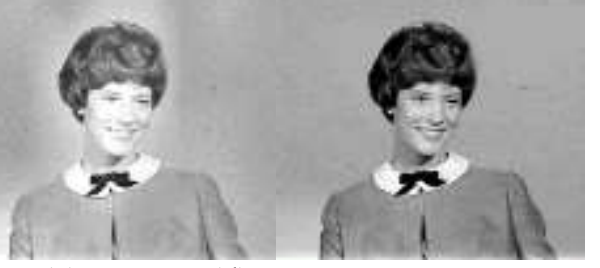

(c) (d)

Figure 6 Enhancement results. (a) Original image; (b) HE; (c)CLAHE ; (d) The proposed method

Figure 6 shows that the details of the output image are handled well and are more in line with human visual characteristics. The enhancement algorithm proposed in this paper achieved good results in terms of enhancing image 
contrast and detail processing. The brightness of the image was appropriately improved in the comparison of Figure 4(a) with Figure 4(c). Image information is also largely preserved, texture is well processed, and noise amplification or other undesirable phenomena did not occur.

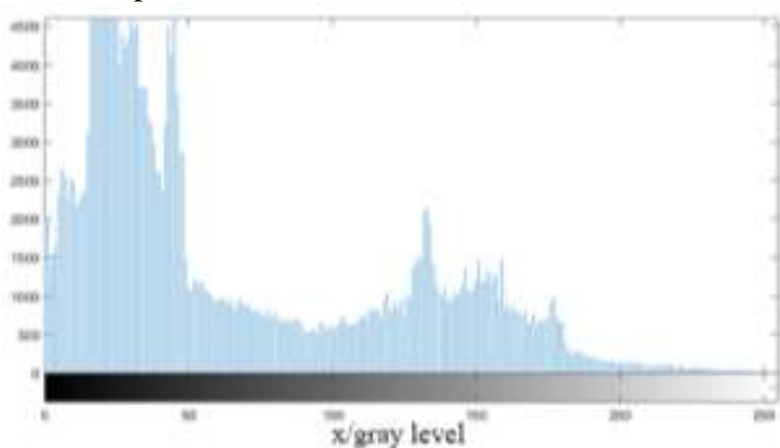

(a)

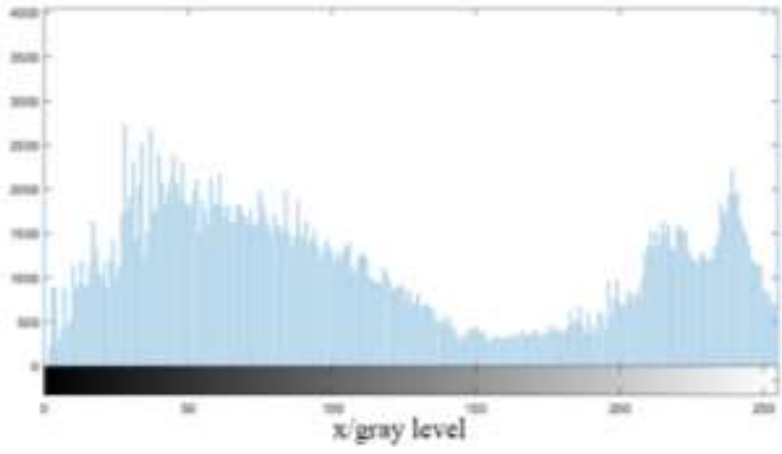

(c)

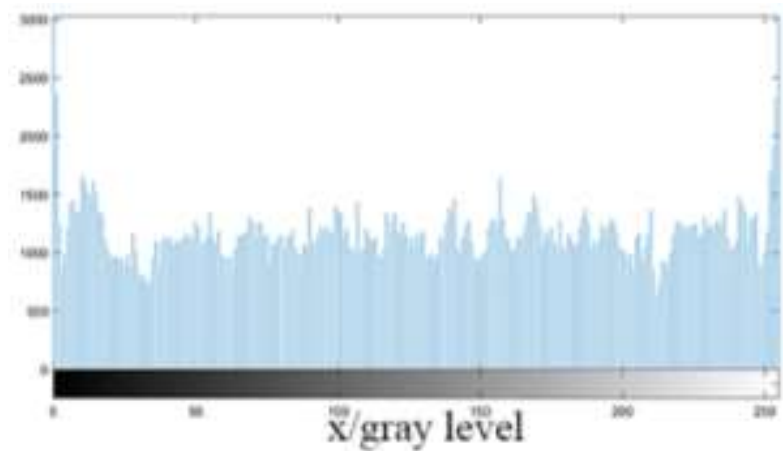

(b)

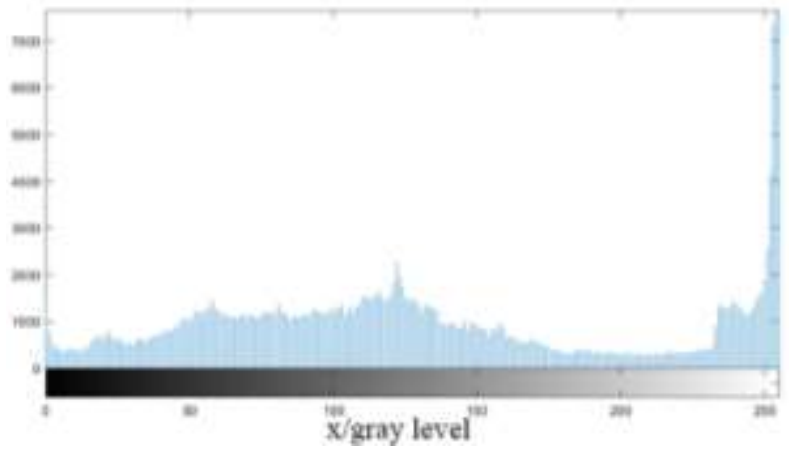

(d)

Figure 7 Histograms of original and enhanced images shown in Figure 4; (a) Original image; (b) HE; (c) CLAHE; (d)

\subsection{Objective Analysis}

the proposed method

The following method used contrast value, the gray average gradient, and the Laplace operator value of the input and output images for comprehensive evaluation.

Image contrast ${ }^{[13]}$ was measured by standard deviation. The larger the standard deviation, the larger the contrast of the image, and the larger the contrast stretch of the image, and the better the image enhancement quality.

$$
\text { contrast }=\sqrt{\sum_{i=0}^{L}\left(x_{i}-\mu\right) \times P\left(x_{i}\right)} .
$$

$x_{i}$ is the grayscale level of an image. $\mu$ is the average brightness of an image. $P\left(x_{i}\right)$ is the probability density when the grayscale level is $x_{i}$.

The average grayscale gradient ${ }^{[14]}(\mathrm{GMG})$ can better reflect the contrast of the image and the change characteristics of texture information. Generally, the larger value of the image, the clearer the image quality is.

$G M G=\frac{1}{(M-1)(N-1)} \sum_{i=1}^{M-1} \sum_{j=1}^{N-1} \sqrt{\frac{W_{i}+W_{j}}{2}}$.

$W_{i}$ is the pixel grayscale value of the input image. $W_{j}$ is the pixel grayscale value of the output image.

The Laplacian ${ }^{14}$ is the differential value in each $3 \times 3$ neighborhood. Generally, the larger the value of the Laplace operator, the clearer the image, and the more distinct the contour.

Lap $=\frac{\sum_{i=2}^{M-1} \sum_{j=2}^{N-1}\left|W(i, j)-W^{\prime}\right|}{(M-2)(N-2)}$.

In the above equation, $W^{i}$ is the sum of the gray values of the $W(i, j)$ neighbor pixels.

The following unit data combined with subjective analysis indicates that this algorithm is effective.

Table 1: Contrast of enhanced images 


\begin{tabular}{|c|c|c|c|}
\hline Image & Fig.4 & Fig.5 & Fig.6 \\
\hline $\begin{array}{c}\text { CLAHE } \\
\text { GMM } \\
\end{array}$ & $\begin{array}{l}14.1007 \\
14.1297 \\
\end{array}$ & $\begin{array}{c}78.4848 \\
144.0793 \\
\end{array}$ & $\begin{array}{l}43.0312 \\
60.3798 \\
\end{array}$ \\
\hline Image & Fig.4 & Fig.5 & Fig.6 \\
\hline $\begin{array}{l}\text { CLAHE } \\
\text { GMM }\end{array}$ & $\begin{array}{l}0.0118 \\
0.0122\end{array}$ & $\begin{array}{l}0.0236 \\
0.0322\end{array}$ & $\begin{array}{l}0.0189 \\
0.0225 \\
\end{array}$ \\
\hline Image & Fig 4 & Fig 5 & Fio 6 \\
\hline $\begin{array}{l}\text { CLAHE } \\
\text { GMM }\end{array}$ & $\begin{array}{l}0.1059 \\
0.1804\end{array}$ & $\begin{array}{l}0.1922 \\
0.3020\end{array}$ & $\begin{array}{l}0.8510 \\
1.0196\end{array}$ \\
\hline
\end{tabular}

\section{CONCLUSION}

A low light image automatic enhancement model based on Gaussian mixture model is proposed in this paper to improve the quality of low-light images. The Gaussian mixture model was solved by constructing a Gaussian mixture model to enhance the image using histogram characteristics. An accelerated convergence EM algorithm based on robustness was also used. The data in the objective analysis showed that the average grayscale gradient and Laplace operator value and contrast of the image increased compared with the existing algorithms. Relative to the algorithms that are currently available, the contrast of the enhanced image increased by nearly $28 \%$, the average grayscale gradient increased by nearly $2.3 \%$, and the Laplace operator value increased by nearly $26 \%$. Subjective analysis and objective data show that the model significantly improves the contrast of the image and does not produce undesirable phenomena, such as saturation and noise amplification. The model preserves the texture details of the image to a large extent, effectively improves the ability of image recognition, and presents an enhancement effect that outperforms the traditional algorithm.

\section{ACKNOWLEDGMENT}

This work was supported by the National Natural Science Foundation of China (61801272); the College Students Innovation and Entrepreneurship Plan (20181043304X06); the SDUT and Zhangdian District Integration Project 2018 (9001/118228)

\section{REFERENCES}

[1] Author, "Title", Chinese Journal of Liquid Crystals and Displays, vol. 28, no. 6, pp. 968-972, 2013.

[2] Author, "Title", Optics and Precision Engineering, vol. 19, no. 3, pp. 2260-2265, 2013.

[3] Author, "Title”, Beijing University of Technology, vol. 13, no. 4, pp. 343-348, 1998.

[4] Author, "Title," Computer Knowledge and Technology, vol. 6, no. 9, pp. 2238-2241, 2010.

[5] Author, "Title", Application Research of Computers, vol. 34, no. 4, pp. 1043-1046, 2016.

[6] Author, "Title", Journal of Optoelectronics • Laser, vol. 24, no. 4, pp. 751-757, 2013.

[7] Author, "Title", Computer Science, vol. 42, no. 6, pp. 241-242, 2015.

[8] Author, "Title”, Beijing Polytechnic University, Beijing, pp. 43-46, 2009.

[9] Author, "Title", China Laser, vol. 41, no. 12, pp. 1-6, 2014.

[10] Author, "Title", University of Chinese Academy of Science Institute of Technical Physics, shanghai, 2015.

[11] Author, "Title", Xi'an University of Electronic Science and Technology, Xian, pp.22-25, 2012.

[12] Author, "Title", Beijing Jiaotong University, pp.39-47, Beijing, 2010.

[13] Author, "Title", IEEE Transaction on Consumer Electronic, vol. 53, no. 3, pp. 1186-1194, 2007.

[14] Author, Title, Publishing House of Electronics Industry, Beijing, 2008.

[15] Author, "Title", Applied Optics, vol. 34, no. 6, pp. 986-989, 2013. 